\section{The effects of medial ventral lesions on avoidance learning'}

JAMES MOTTIN and JOHN GAITO, York University, Toronto, Ontario, Canada

Lesions associated with the medial ventral cortex of rats impaired one-way shockavoidance learning. These results, combined with previous neurochemical experiments, suggest that this area contributes to this learning.

Results of previous experiments using ratios of nucleic acids and proteins suggested that medial ventral (MV) tissue of the rat brain was a likely contributor to learning in shock-avoidance conditioning (Gaito, Mottin, \& Davison, 1968) and in a water-maze task (Gaito, Davison, \& Mottin, 1969). This tissue, one of numerous brain sections analyzed, contained both cortical and subcortical portions and was circumscribed by a horizontal cut along the rhinal fissure, a dorsal-ventral cut along the ventral depression left by the middle cerebral artery, and a similar cut approximately midway between the middle cerebral artery stereotaxic instrument using bilateral coordinates predetermined by a stereotaxic "tear-down" of other animals.

With the animal's skull leveled in the stereotaxic instrument, the coordinates were relative to Ear-Bar-Zero coordinates. For Experiment 1 the MV lesion was made $5.0 \mathrm{~mm}$ anteriorly of EBZ, $4.0 \mathrm{~mm}$ left and right of EBZ, and $0.0 \mathrm{~mm}$ above $\mathrm{EBZ}$. The MD lesion for Experiment 1 was achieved by placing the electrode on the surface of the dorsal cortex at the anterior and lateral coordinates for MV lesions. Bilateral lesions were made with anodal current of $1 \mathrm{~mA}$ for $20 \mathrm{sec}$ for both MD and MV lesions. Ss in the sham subgroup merely had the electrodes lowered to the specific medial ven tral cortex (MV) coordinates without the application of current. Following bilateral lesions or sham treatment, the scalp was closed with sutures.

The electrodes used were made from blunt ended steel wire having a diameter of .0152 in. An Epoxylite insulation covered the electrode surface which was projected into the animal, bringing the total diameter groups using a Littermates by Treatments ANOVA design.

\section{EXPERIMENT 2} that MV tissue was not necessary in shock-avoidance conditioning. However, the size of the lesion was minimal; a major portion of MV remained which could have contributed to the conditioning process. Thus in Experiment 2 the MD group was eliminated and the size of the lesion in MV increased (Fig. 1D).

The lesioning end of the electrode was free from insulation for a height of .020 in. and a current of $3 \mathrm{~mA}$ for $30 \mathrm{sec}$ was used. One millimeter was added to the anterior and lateral coordinates from Experiment 1 while the dorsal coordinate was $1.5 \mathrm{~mm}$ above EBZ.

The Ss consisted of nine pairs of male littermate rats of the Wistar strain. The weights and ages were the same as in Experiment 1. Shock avoidance procedures were the same as in Experiment 1. The three behavioral dependent variables showed
The results of Experiment 1 suggested 


\section{The effects of medial ventral lesions on avoidance learning'}

JAMES MOTTIN and JOHN GAITO, York University, Toronto, Ontario, Canada

Lesions associated with the medial ventral cortex of rats impaired one-way shockavoidance learning. These results, combined with previous neurochemical experiments, suggest that this area contributes to this learning.

Results of previous experiments using ratios of nucleic acids and proteins suggested that medial ventral (MV) tissue of the rat brain was a likely contributor to learning in shock-avoidance conditioning (Gaito, Mottin, \& Davison, 1968) and in a water-maze task (Gaito, Davison, \& Mottin, 1969). This tissue, one of numerous brain sections analyzed, contained both cortical and subcortical portions and was circumscribed by a horizontal cut along the rhinal fissure, a dorsal-ventral cut along the ventral depression left by the middle cerebral artery, and a similar cut approximately midway between the middle cerebral artery stereotaxic instrument using bilateral coordinates predetermined by a stereotaxic "tear-down" of other animals.

With the animal's skull leveled in the stereotaxic instrument, the coordinates were relative to Ear-Bar-Zero coordinates. For Experiment 1 the MV lesion was made $5.0 \mathrm{~mm}$ anteriorly of EBZ, $4.0 \mathrm{~mm}$ left and right of EBZ, and $0.0 \mathrm{~mm}$ above $\mathrm{EBZ}$. The MD lesion for Experiment 1 was achieved by placing the electrode on the surface of the dorsal cortex at the anterior and lateral coordinates for MV lesions. Bilateral lesions were made with anodal current of $1 \mathrm{~mA}$ for $20 \mathrm{sec}$ for both MD and MV lesions. Ss in the sham subgroup merely had the electrodes lowered to the specific medial ven tral cortex (MV) coordinates without the application of current. Following bilateral lesions or sham treatment, the scalp was closed with sutures.

The electrodes used were made from blunt ended steel wire having a diameter of .0152 in. An Epoxylite insulation covered the electrode surface which was projected into the animal, bringing the total diameter groups using a Littermates by Treatments ANOVA design.

\section{EXPERIMENT 2} that MV tissue was not necessary in shock-avoidance conditioning. However, the size of the lesion was minimal; a major portion of MV remained which could have contributed to the conditioning process. Thus in Experiment 2 the MD group was eliminated and the size of the lesion in MV increased (Fig. 1D).

The lesioning end of the electrode was free from insulation for a height of .020 in. and a current of $3 \mathrm{~mA}$ for $30 \mathrm{sec}$ was used. One millimeter was added to the anterior and lateral coordinates from Experiment 1 while the dorsal coordinate was $1.5 \mathrm{~mm}$ above EBZ.

The Ss consisted of nine pairs of male littermate rats of the Wistar strain. The weights and ages were the same as in Experiment 1. Shock avoidance procedures were the same as in Experiment 1. The three behavioral dependent variables showed
The results of Experiment 1 suggested 